\title{
Local nonsimilarity method for the two-phase boundary layer in mixed convection laminar film condensation
}

\author{
Y. Liao $\cdot$ S. Guentay $\cdot$ K. Vierow
}

Received: 4 December 2009/Accepted: 28 February 2010/Published online: 14 March 2010

(C) Springer-Verlag 2010

\begin{abstract}
The two-phase boundary layer in laminar film condensation was solved by Koh for the free convection regime and forced convection regime using the similarity method. But the problem on mixed convection does not admit similarity solutions. The current work develops a local nonsimilarity method for the full spectrum of mixed convection, with a generic boundary layer formulation reduced to two specific cases mathematically identical to Koh's formulations on the two limiting cases for either free or forced convection. Other solution methods for mixed convection in the literature are compared and critically evaluated to ensure a high level of accuracy in the current method. The current solution is used to extend Fujii's correlation for mixed convection to the region where the energy convection effect is significant but has been hitherto neglected. The modified Fujii correlation provides a practical engineering tool for evaluating laminar film condensation with a mixed convection boundary layer.
\end{abstract}

\section{List of symbols}

$$
\begin{array}{ll}
c_{L} & {\left[g\left(\rho_{L}-\rho_{v}\right) /\left(4 v_{L}^{2} \rho_{L}\right)\right]^{1 / 4}\left(\mathrm{~m}^{-3 / 4}\right)} \\
c_{p} & \text { Specific heat }\left(\mathrm{J} \mathrm{kg}^{-1} \mathrm{~K}^{-1}\right) \\
c_{v} & {\left[g /\left(4 v_{v}^{2}\right)\right]^{1 / 4}\left(\mathrm{~m}^{-3 / 4}\right)} \\
f & \text { Vapor dimensionless stream function } \\
F & \text { Liquid dimensionless stream function } \\
g & \text { Gravity constant }\left(\mathrm{m} \mathrm{s}^{-2}\right) \\
h & q_{w} / \Delta T\left(\mathrm{~W} \mathrm{~m}^{-2} \mathrm{~K}^{-1}\right)
\end{array}
$$

Y. Liao $(\bowtie) \cdot$ S. Guentay

Laboratory for Thermal-Hydraulics, Paul Scherrer Institute (PSI), 5232 Villigen, Switzerland

e-mail: Yehong.liao@psi.ch

K. Vierow

Department of Nuclear Engineering, Texas A\&M University,

MS 3133, College Station, TX 77843, USA $h_{f g} \quad$ Latent heat $\left(\mathrm{J} \mathrm{kg}^{-1}\right)$

$H \quad$ Condensation number, Eq. 19

$k \quad$ Thermal conductivity $\left(\mathrm{W} \mathrm{m}^{-1} \mathrm{~K}^{-1}\right.$ )

$N u_{x} \quad h x / k_{L}$

Pr Prandtl number

$q \quad$ Local heat flux $\left(\mathrm{W} \mathrm{m}^{-2}\right)$

$R e_{x} \quad u_{\infty} x / v_{L}$

$T \quad$ Temperature (K)

$\Delta T \quad T_{\infty}-T_{w}(\mathrm{~K})$

$u \quad$ Longitudinal velocity $\left(\mathrm{m} \mathrm{s}^{-1}\right)$

$u_{\infty} \quad$ Vapor bulk velocity $\left(\mathrm{m} \mathrm{s}^{-1}\right)$

$v$ Transverse velocity $\left(\mathrm{m} \mathrm{s}^{-1}\right)$

$x \quad$ Longitudinal coordinate (m)

$y \quad$ Transverse coordinate (m)

$z \quad g x / u_{\infty}^{2}$

\section{Greek symbols}

$\alpha$ Correction factor, Eq. 26

$\delta \quad$ Liquid film thickness (m)

$\eta \quad$ Pseudo-similarity variable

$\mu$ Absolute viscosity $\left(\mathrm{kg} \mathrm{s}^{-1} \mathrm{~m}^{-1}\right)$

$v$ Kinematic viscosity $\left(\mathrm{m}^{2} \mathrm{~s}^{-1}\right)$

$\theta \quad\left(T-T_{\infty}\right) /\left(T_{w}-T_{\infty}\right)$

$\rho$ Density $\left(\mathrm{kg} \mathrm{m}^{-3}\right)$

$\omega \quad\left[\rho_{L} \mu_{L} /\left(\rho_{v} \mu_{v}\right)\right]^{1 / 2}$

$\xi \quad$ Mixed convection parameter, Eq. 1

$\psi$ Stream function

\section{Subscripts}

$0 \quad$ Wall

$i$ Two-phase interface

$L \quad$ Liquid phase

$v \quad$ Vapor phase

$w \quad$ Wall

$\infty \quad$ Vapor bulk 


\section{Introduction}

Since Nusselt's publication of the pioneering work on laminar film condensation [1], an abundance of research has been carried out to study the condensation phenomena in different convection regimes $[2,3]$. In the free convection regime, the liquid film is mainly driven by the gravity force. While in the forced convection regime, the liquid film is mainly driven by the interfacial shear force exerted by the flowing vapor. In between is the mixed convection regime, where the liquid film is driven by both the gravity and interfacial shear forces having almost the same order of magnitude. The analytical closed-form solution of laminar condensation was given by Nusselt for the free convection regime and by Fujii for the forced and mixed convection regimes [4]. However, these solutions were derived from the momentum integral approach which neglects the liquid inertia and energy convection effect. To account for this effect, a few studies have proposed various correction factors used with the afore-mentioned closed formed solution, but so far only for the free convection regime [5-7]. The current work develops the correction factors for the liquid inertia and energy convection effect for the entire mixed convection regime using the numerical solution of the two-phase boundary layer equations for laminar film condensation in the entire mixed convection regime.

The two-phase boundary layer formulation was introduced into studies of free convection film condensation by Sparrow and Gregg [8] and Koh et al. [9]. Koh [10] further applied the boundary layer formulation to study forced convection film condensation. The similarity method was used in Sparrow and Koh's pioneering work on either free or forced convection, but the mixed convection problem dealt with by the current work does not admit similarity solutions. Either the finite difference method $[11,12]$ or the local nonsimilarity method [13] can be used to solve the two-phase boundary layer equations for mixed convection laminar condensation. However, none of these previous studies [11-13] has been able to demonstrate in a rigorous way that their solutions at the two limits of mixed convection coincide with the known solutions given by Koh on either free convection [9] or forced convection [10]. This is a nontrivial problem to investigate, because only if the limit solutions are correct can a high level of confidence in the solutions in between be attained. This is also of theoretical interest since such a solution provides a complete formulation for mixed convection consistent with those two end points already fixed by Koh's work $[9,10]$.

The current work uses the local nonsimilarity method [14] to solve the mixed convection two-phase boundary layer equations, and tries to fulfill two complementary objectives. First, the local nonsimilarity method is applied with a generic boundary layer formulation which can be demonstrated to be mathematically consistent with Koh's formulations for the limiting cases of either free or forced convection. Second, the current work presents the solution for the full spectrum of mixed convection and compares with other solution methods in the literature to achieve a high level of accuracy. As a result, the current numerical solution is used to extend Fujii's correlation [4] for mixed convection laminar film condensation to the region where the energy convection effect is substantial but has been hitherto neglected.

\section{Boundary layer formulation}

Koh et al.'s [9] formulation of laminar condensation for free convection is summarized below, as this is the starting point of the current work: stagnant bulk vapor at a saturation temperature of $T_{\infty}$ condenses along an isothermal vertical plate at a temperature of $T_{w}$; the two-phase boundary layer equations were formulated in the coordinate system with the longitudinal coordinate $(x)$ pointing downward and the transverse coordinate $(y)$ pointing normal to the plate. The following analysis of the mixed convection condensation process is governed by the same set of boundary layer equations with the same coordinate system presented by Koh et al. [9] and it is not necessary to repeat them here. The major difference between the current work and Koh et al.'s [9] is that, in free convection condensation studied by Koh et al. [9], the vapor bulk was stagnant so that the similarity solutions were achieved, while in mixed convection condensation studied in the current work, vapor flows downward with a bulk velocity $u_{\infty}$ not necessarily restricted to zero so that the condensation process is in the mixed convection regime and the problem becomes nonsimilar.

\subsection{Local nonsimilarity transformation for the mixed convection boundary layer equations}

In addition to the dimensionless parameters $\left(H, \omega\right.$ and $\left.P r_{L}\right)$ governing the condensation processes of either free or forced convection, a mixed convection parameter is introduced herein for the local nonsimilarity method and later used to correlate a formula for laminar film condensation in the entire mixed convection regime:

$\xi=\frac{1}{1+\left[u_{\infty}^{2} /(g x)\right]^{1 / 4}}$

This mixed convection parameter indicates the relative importance of free convection $(g x)$ over forced convection $\left(u_{\infty}^{2}\right)$. At the leading edge of the plate, forced convection dominates over free convection $u_{\infty}^{2} \gg g x$, and therefore $\xi \rightarrow 0$. At the far field of the plate, free convection 
dominates over forced convection $g x \gg u_{\infty}^{2}$, and therefore $\xi \rightarrow 1$. In between is the mixed convection regime: $0<\xi<1$.

For the liquid conservation equations, a pseudo-similarity variable, $\eta_{L}=c_{L} y /\left(x^{1 / 4} \xi\right)$, and a stream function, $\psi_{L}=4 v_{L} c_{L} x^{3 / 4} F\left(\eta_{L}, \xi\right) / \xi$, are introduced herein to transform the liquid phase velocity components in the $(x, y)$ to the $\left(\xi, \eta_{L}\right)$ coordinates using the chain rule for derivatives as:

$u_{L}=\frac{\partial \psi_{L}}{\partial y}=4 v_{L} c_{L}^{2} x^{1 / 2} F^{\prime} / \xi^{2}$

$v_{L}=-\frac{\partial \psi_{L}}{\partial x}=-\frac{4 v_{L} c_{L}}{x^{1 / 4} \xi}\left[\frac{2+\xi}{4} F+x F^{\prime} \frac{\partial \eta_{L}}{\partial x}+x \frac{d \xi}{d x} \frac{\partial F}{\partial \xi}\right]$

$\frac{\partial u_{L}}{\partial y}=4 v_{L} c_{L}^{3} x^{1 / 4} F^{\prime \prime} / \xi^{3}$

which are used herein to transform the liquid momentum and energy equations to:

$$
\begin{aligned}
& F^{\prime \prime \prime}+(2+\xi) F F^{\prime \prime}-2 \xi\left(F^{\prime}\right)^{2}+\xi^{4} \\
& \quad=\xi(1-\xi)\left(F^{\prime} \frac{\partial F^{\prime}}{\partial \xi}-F^{\prime \prime} \frac{\partial F}{\partial \xi}\right) \\
& \frac{\theta^{\prime \prime}}{P r_{L}}+(2+\xi) F \theta^{\prime}=\xi(1-\xi)\left(F^{\prime} \frac{\partial \theta}{\partial \xi}-\theta^{\prime} \frac{\partial F}{\partial \xi}\right)
\end{aligned}
$$

where the primes denote differentiation with respect to the liquid pseudo-similarity variable $\left(\eta_{L}\right)$.

For the vapor conservation equations, since vapor is assumed at the saturation temperature, only the momentum equation is considered. Analogous to the liquid phase, a pseudo-similarity variable, $\eta_{v}=c_{v}[y-\delta(x)] /\left(x^{1 / 4} \xi\right)$, and a stream function, $\psi_{v}=4 v_{v} c_{v} x^{3 / 4} f\left(\eta_{v}, \xi\right) / \xi$, are introduced herein to transform the vapor phase velocity components to:

$$
\begin{aligned}
& u_{v}=\frac{\partial \psi_{v}}{\partial y}=4 v_{v} c_{v}^{2} x^{1 / 2} f^{\prime} / \xi^{2} \\
& v_{v}=-\frac{\partial \psi_{v}}{\partial x}=-\frac{4 v_{v} c_{v}}{x^{1 / 4} \xi}\left[\frac{2+\xi}{4} f+x f^{\prime} \frac{\partial \eta_{v}}{\partial x}+x \frac{d \xi}{d x} \frac{\partial f}{\partial \xi}\right] \\
& \frac{\partial u_{v}}{\partial y}=4 v_{v} c_{v}^{3} x^{1 / 4} f^{\prime \prime} / \xi^{3}
\end{aligned}
$$

which are used herein to transform the vapor momentum equation to:

$f^{\prime \prime \prime}+(2+\xi) f f^{\prime \prime}-2 \xi\left(f^{\prime}\right)^{2}=\xi(1-\xi)\left(f^{\prime} \frac{\partial f^{\prime}}{\partial \xi}-f^{\prime \prime} \frac{\partial f}{\partial \xi}\right)$

where the primes denote differentiation with respect to the vapor pseudo-similarity variable $\left(\eta_{v}\right)$.

The following subsections present the derivation of nine new closure equations in (11) through (19) to solve the transformed liquid and vapor boundary layer equations in (5), (6) and (10).

\subsection{Liquid and vapor interfacial continuity relations}

The velocity components listed in Eqs. $2-4$ for the liquid phase and in Eqs. 7-9 for the vapor phase are evaluated at $y=\delta(x)$ to derive herein the closure equations for continuity of velocity, balance of mass and balance of tangential shear at the two-phase interface.

Upon evaluating Eqs. 2 and 7 at the two-phase interface: $(u)_{v, i}=(u)_{L, i}$, the continuity of the interfacial longitudinal velocity can be expressed using the transformed variables as:

$f_{i}^{\prime}=\left(1-\rho_{v} / \rho_{L}\right)^{1 / 2} F_{i}^{\prime}$

The mass of vapor transferred to the interface must be equal to the mass of liquid transferred from the interface to the condensate film [9]: $[\rho(v-u d \delta / d x)]_{v, i}=[\rho(v-$ $u d \delta / d x)]_{L, i}$. Using the transformed velocity components in Eqs. 2, 3, 7 and 8, the mass balance written in the new coordinate system is derived herein as:

$$
\begin{aligned}
(2+\xi) f_{i}+\xi(1-\xi)\left(\frac{\partial f}{\partial \xi}\right)_{i}=\omega\left(1-\rho_{v} / \rho_{L}\right)^{1 / 4} \\
\quad \times\left[(2+\xi) F_{i}+\xi(1-\xi)\left(\frac{\partial F}{\partial \xi}\right)_{i}\right]
\end{aligned}
$$

The balance of tangential shear at the two-phase interface can be written by: $(\mu \partial u / \partial y)_{v, i}=(\mu \partial u / \partial y)_{L, i}$. Upon substituting the transformed velocity components, it is obtained herein that:

$f_{i}^{\prime \prime}=\omega\left(1-\rho_{v} / \rho_{L}\right)^{3 / 4} F_{i}^{\prime \prime}$

\subsection{Boundary conditions}

At the wall, the liquid phase boundary conditions, $v_{L 0}=0$, $u_{L 0}=0$, and $T_{L 0}=T_{w}$ are rewritten herein in terms of the transformed variables as:

$$
\begin{aligned}
& F_{\eta_{L}=0}=-\xi(1-\xi)\left(\frac{\partial F}{\partial \xi}\right)_{\eta_{L}=0} /(2+\xi) \\
& F_{\eta_{L}=0}^{\prime}=0 \\
& \theta_{\eta_{L}=0}=1
\end{aligned}
$$

At the liquid-vapor interface, the liquid phase boundary condition, $T_{L i}=T_{\infty}$ is expressed as

$\theta_{\eta_{L}=\eta_{i}}=0$

Far away from the interface, the vapor phase boundary condition, $u_{v \infty}=u_{\infty}$, and the definition of $\xi$ are inserted into Eq. 7 to arrive herein at

$f_{\eta_{v}=\infty}^{\prime}=(1-\xi)^{2} / 2$ 


\subsection{Interfacial energy balance}

The latent heat of condensation is balanced by the overall heat transfer from the liquid-vapor interface to the condensate film [9]: $[\rho(v-u d \delta / d x)]_{L, i} h_{f g}=k_{L}(\partial T / \partial y)_{L, i}$. Using the transformed velocity components, the energy balance in the new coordinate system is derived herein as:

$H=\frac{c_{p L} \Delta T}{\operatorname{Pr}_{L} h_{f g}}=-\frac{(2+\xi) F_{i}+\xi(1-\xi)\left(\frac{\partial F}{\partial \xi}\right)_{i}}{\theta_{i}^{\prime}}$

\subsection{Heat transfer at the wall}

Heat transfer rate at the wall is expressed as: $q=k_{L}(\partial T /$ $\partial y)_{w}$, where using the definitions of the transformed temperature variable $(\theta)$ and the mixed convection parameter $(\xi)$, heat transfer at the wall in terms of the Nusselt number is derived herein as:

$$
\frac{N u_{x}}{R e_{x}^{1 / 2}}=-\frac{\theta_{0}^{\prime}}{\sqrt{2}(1-\xi)}\left(1-\frac{\rho_{v}}{\rho_{L}}\right)^{1 / 4}
$$

\section{Local nonsimilarity solution of boundary layer equations}

The full spectrum of mixed convection can be studied by varying the mixed convection parameter $\xi$ continuously from 0 to 1 in the current transformed boundary layer equations in (5), (6) and (10). The two limiting cases of free $(\xi=1)$ and forced $(\xi=0)$ convection have been solved by Koh's work [9, 10]. This section first discusses the solutions for the two limiting cases using the current formulation in light of the known solutions in Koh's pioneering work $[9,10]$, and then presents the solution for the gap in between.

\subsection{The limiting case of free convection $(\xi \rightarrow 1)$}

Herein it is proved that the current formulation coincides with Koh et al.'s work [9] on free convection as $\xi \rightarrow 1\left(u_{\infty}\right.$ becomes small and/or $x$ becomes large). For the limiting case of $\xi \rightarrow 1$, the right-hand side of the transformed equations in (5), (6) and (10) reduces to zero, and it is immediately recognized that the current reduced transformed equations are identical to their counterparts in Koh's work (Eqs. 14, 15, 19 in [9]). It is not difficult to confirm that the nine closure equations in (11)-(19) are also identical to their counterparts in Koh's work: Eqs. 11-19 in the current work corresponding, respectively, to Eqs. 21, 23, $25,26,26,29,30,28,32$ in [9], when $\xi \rightarrow 1$ and $\rho_{v} \ll \rho_{L}$. Therefore, the current boundary layer formulation is demonstrated to have the limit solution for free convection identical to that in Koh's work [9].

\subsection{The limiting case of forced convection $(\xi \rightarrow 0)$}

Herein it is proved that the current formulation coincides with Koh's work [10] on forced convection as $\xi \rightarrow 0$ ( $x$ becomes small and/or $u_{\infty}$ becomes large). When $\xi \rightarrow 0$, the right-hand side of the current transformed equations in (5), (6) and (10) reduces to zero. The reduced liquid momentum equation is listed below to compare with its counterpart in Koh's work [10]:

$F^{\prime \prime \prime}+2 F F^{\prime \prime}=0$

At first glance, the current transformed liquid momentum equation (21) looks different from its counterpart in Koh's work (Eq. 1 of [10]). Actually, they are equivalent if the difference in the respective scaling factors for the similarity variable $\left(\eta_{L}\right)$ and the dimensionless stream function $(F)$ used in carrying out the transformation of the conservation equation is carefully taken into account. The assumption $\rho_{v} \ll \rho_{L}$ is used in the following proof.

From the definition of $\xi$, it follows that $\xi=$ $\left(g x / u_{\infty}^{2}\right)^{1 / 4}(1-\xi)$, which is substituted into the definition of $\eta_{L}$ to obtain $\eta_{L}=c_{L} y /\left[x^{1 / 4}\left(g x / u_{\infty}^{2}\right)^{1 / 4}(1-\xi)\right]$, or $\eta_{L}=$ $(y / x) \sqrt{u_{\infty} x / v_{L}} /[\sqrt{2}(1-\xi)]$. From the definition of the similarity variable used by Koh, when $\xi \rightarrow 0$ it is obtained that

$\eta_{L}=\eta_{L, \mathrm{Koh}} / \sqrt{2}$

where $\eta_{L, \text { Koh }}$ denotes the similarity variable used by Koh [10].

Upon substituting $\xi=\left(g x / u_{\infty}^{2}\right)^{1 / 4}(1-\xi)$ into the definition of $\psi_{L}$, the liquid-phase stream function is expressed as $\psi_{L}=2 \sqrt{2} \sqrt{v_{L} u_{\infty} x} F /(1-\xi)$. From the definition of the stream function used by Koh and equating the stream functions $\psi_{L}=\psi_{L, \text { Koh }}$, it follows that when $\xi \rightarrow 0$,

$F=F_{\mathrm{Koh}} /(2 \sqrt{2})$

where $\psi_{L, \text { Koh }}$ and $F_{\text {Koh }}$ denote, respectively, the stream function and the dimensionless stream function used by Koh [10].

Substituting $\eta_{L}$ in (22) and $F$ in (23) into $F^{\prime}=d F / d \eta_{L}$, it leads to $F^{\prime}=\left(d F_{\mathrm{Koh}} / d \eta_{L, \mathrm{Koh}}\right) / 2=F_{\mathrm{Koh}}^{\prime} / 2$. Similarly, it can be shown that $F^{\prime \prime}=F_{\mathrm{Koh}}^{\prime \prime} / \sqrt{2}$ and $F^{\prime \prime \prime}=F_{\mathrm{Koh}}^{\prime \prime \prime}$. Upon substituting $F, F^{\prime \prime}, F^{\prime \prime \prime}$ into the transformed liquid momentum equation in (21), it follows that $F_{\mathrm{Koh}}^{\prime \prime \prime}+$ $F_{\mathrm{Koh}} F_{\text {Koh }}^{\prime \prime} / 2=0$, which is exactly the formulation used in Koh's work for forced convection (Eq. 1 of [10]). In a similar way, it can also be shown that the other transformed conservation equations are equivalent to their counterparts 
in Koh's work [10]. It is rather lengthy but not difficult to show that the current closure equations are also equivalent to their counterparts in Koh's work [10]. Therefore, the current boundary layer formulation is shown to have the limit solution for forced convection identical to that in Koh's work [10].

\subsection{Local nonsimilarity solution for the entire mixed convection regime $(0 \leq \xi \leq 1)$}

When $\xi \rightarrow 0$ or $\xi \rightarrow 1$, the problems admit similarity solutions. The right-hand side of the current transformed equations in (5), (6) and (10) is equal to 0 , and the equations in (5), (6) and (10) can be reduced to a system of eight-first-order ordinary differential equations (ODEs) involving eight unknown variables (which include three variables for the third-order ODE about $F$, two for the second-order ODE about $\theta$, and three for the third-order ODE about $f$ ). In addition to these eight unknown variables, there is one unknown variable, $\eta_{i}$, in the closure equations. On the other hand, there are altogether nine closure equations: Eqs. 11 through 19. Therefore, the system prescribed by Eqs. 5, 6 and 10 is closed.

When $0<\xi<1$, the solution of the current transformed equations becomes more complicated due to additional unknown variables emerging from the right-hand side of Eqs. 5, 6 and 10 about the first-order differentiation with respect to $\xi: \partial F / \partial \xi, \partial \theta / \partial \xi, \partial f / \partial \xi$. But they can be solved using the local nonsimilarity method [14]. The main idea of the local nonsimilarity method is to reduce a set of partial differential equations to a set of ODEs that is easier to solve. In the first-level approximation of the local nonsimilarity method, the first-order differentiations with respect to $\xi$ are truncated, and therefore the equations are solved as in the similarity solution discussed at the beginning of this subsection. In the second-level approximation, when introducing new variables: $P=\partial F / \partial \xi$, $Q=\partial \theta / \partial \xi, p=\partial f / \partial \xi$, and differentiating both sides of Eqs. 5, 6 and 10 with respect to the mixed convection parameter $\xi$, the resulting equations become ODEs if the truncation errors due to the terms involving differentiation with respect to $\xi$ with orders equal to or greater than two are neglected. A higher level of accuracy can be achieved by continuing this process, to retain the terms involving the second-order differentiation with respect to $\xi$ while truncate the terms with differentiation orders equal to or greater than three (the third-level approximation).

The current work proceeds with the local nonsimilarity method from the first-level through the third-level approximation and will compare the solutions of different levels of approximation to investigate the asymptotical behavior of the local nonsimilarity method in Table 1 .

When carrying out the local nonsimilarity method in this way for the second-level approximation, the auxiliary

Table 1 Comparison of heat transfer results among various solution methods: $\operatorname{Pr}_{L}=10$ and $\omega=10$

\begin{tabular}{|c|c|c|c|c|c|c|c|}
\hline \multirow[t]{3}{*}{$H$} & \multirow[t]{3}{*}{$\xi$} & \multirow[t]{3}{*}{$z$} & \multicolumn{5}{|l|}{$N u_{x} / R e_{x}^{1 / 2}$} \\
\hline & & & \multicolumn{3}{|c|}{ Current work (local nonsimilarity) } & \multirow[t]{2}{*}{ Shu and Wilks [12] } & \multirow[t]{2}{*}{ Fujii and Uehara [4] } \\
\hline & & & 1st level & 2nd level & 3rd level & & \\
\hline \multirow[t]{10}{*}{0.008191} & 0.0 & 0.0 & 1.076 & 1.076 & 1.076 & 1.076 & 1.069 \\
\hline & 0.401 & 0.2 & 1.536 & 1.695 & 1.723 & 1.690 & 1.650 \\
\hline & 0.500 & 1.0 & 2.286 & 2.426 & 2.442 & 2.410 & 2.375 \\
\hline & 0.543 & 2.0 & 2.724 & 2.858 & 2.872 & 2.852 & 2.810 \\
\hline & 0.599 & 5.0 & 3.437 & 3.573 & 3.584 & 3.568 & 3.522 \\
\hline & 0.640 & 10 & 4.099 & 4.238 & 4.246 & 4.234 & 4.184 \\
\hline & 0.701 & 30 & 5.420 & 5.564 & 5.569 & 5.550 & 5.503 \\
\hline & 0.736 & 60 & 6.465 & 6.611 & 6.614 & 6.608 & 6.543 \\
\hline & 0.760 & $1 \mathrm{e} 2$ & 7.360 & 7.507 & 7.510 & 7.493 & 7.434 \\
\hline & 0.909 & $1 \mathrm{e} 4$ & 23.570 & 23.703 & 23.703 & 23.703 & 23.505 \\
\hline \multirow[t]{2}{*}{0.05} & 0.0 & 0.0 & 0.698 & 0.698 & 0.698 & 0.698 & 0.663 \\
\hline & 0.909 & $1 \mathrm{e} 4$ & 15.800 & 15.901 & 15.901 & 15.9 & 15.0 \\
\hline \multirow[t]{2}{*}{0.1} & 0.0 & 0.0 & 0.630 & 0.630 & 0.630 & 0.630 & 0.585 \\
\hline & 0.909 & 1e4 & 13.985 & 14.099 & 14.099 & 14.1 & 12.6 \\
\hline \multirow[t]{2}{*}{0.2} & 0.0 & 0.0 & 0.596 & 0.596 & 0.596 & 0.596 & 0.537 \\
\hline & 0.909 & 1e4 & 12.736 & 12.867 & 12.868 & 12.8 & 10.6 \\
\hline \multirow[t]{2}{*}{0.5} & 0.0 & 0.0 & 0.586 & 0.586 & 0.586 & 0.586 & 0.503 \\
\hline & 0.909 & 1e4 & 11.795 & 11.959 & 11.961 & 11.9 & 8.4 \\
\hline
\end{tabular}


equations about $P, Q, p$ are derived herein by differentiating both sides of Eqs. 5, 6 and 10 with respect to $\xi$ as:

$$
\begin{aligned}
P^{\prime \prime \prime} & +(2+\xi) F P^{\prime \prime}+\xi(1-\xi) P P^{\prime \prime}-(1+2 \xi) F^{\prime} P^{\prime} \\
- & \xi(1-\xi)\left(P^{\prime}\right)^{2}+(3-\xi) F^{\prime \prime} P+F F^{\prime \prime} \\
- & 2\left(F^{\prime}\right)^{2}+4 \xi^{3}=\xi(1-\xi)\left(F^{\prime} \frac{\partial^{2} F^{\prime}}{\partial \xi^{2}}-F^{\prime \prime} \frac{\partial^{2} F}{\partial \xi^{2}}\right) \\
\frac{Q^{\prime \prime}}{P r_{L}}+ & (2+\xi) F Q^{\prime}+\xi(1-\xi) P Q^{\prime}-(1-2 \xi) F^{\prime} Q \\
& -\xi(1-\xi) P^{\prime} Q+(3-\xi) \theta^{\prime} P+F \theta^{\prime} \\
= & \xi(1-\xi)\left(F^{\prime} \frac{\partial^{2} \theta}{\partial \xi^{2}}-\theta^{\prime} \frac{\partial^{2} F}{\partial \xi^{2}}\right) \\
p^{\prime \prime \prime}+ & (2+\xi) f p^{\prime \prime}+\xi(1-\xi) p p^{\prime \prime}-(1+2 \xi) f^{\prime} p^{\prime} \\
- & \xi(1-\xi)\left(p^{\prime}\right)^{2}+(3-\xi) f^{\prime \prime} p \\
& +f f^{\prime \prime}-2\left(f^{\prime}\right)^{2}=\xi(1-\xi)\left(f^{\prime} \frac{\partial^{2} f^{\prime}}{\partial \xi^{2}}-f^{\prime \prime} \frac{\partial^{2} f}{\partial \xi^{2}}\right)
\end{aligned}
$$

With the second-level approximation, the second-order differentiations with respect to $\xi$ on the right-hand side of the equations in (24) are truncated, therefore the transformed conservation equations in (5), (6) and (10) as well as the auxiliary equations in (24) are reduced to ODEs.

A higher level of accuracy can be achieved with the third-level approximation to retain the terms on the righthand side of the equations in (24). While introducing new variables to replace the terms on the right-hand side of the equations in (24) about the second-order differentiations with respect to $\xi: R=\partial^{2} F / \partial \xi^{2}, S=\partial^{2} \theta / \partial \xi^{2}, r=\partial^{2} f / \partial \xi^{2}$, and differentiating both sides of the equations in (24) with respect to $\xi$, the auxiliary equations about $R, S, r$ are derived herein:

$$
\begin{aligned}
& R^{\prime \prime \prime}+(2+\xi) F R^{\prime \prime}+\xi(1-\xi) P R^{\prime \prime}-2 F^{\prime} R^{\prime}-3 \xi(1-\xi) P^{\prime} R^{\prime} \\
& +(4-3 \xi) F^{\prime \prime} R+2 \xi(1-\xi) P^{\prime \prime} R+2 F P^{\prime \prime}+2(3-\xi) P P^{\prime \prime} \\
& \quad-6 F^{\prime} P^{\prime}-2\left(P^{\prime}\right)^{2}+12 \xi^{2}=\xi(1-\xi)\left(F^{\prime} \frac{\partial^{3} F^{\prime}}{\partial \xi^{3}}-F^{\prime \prime} \frac{\partial^{3} F}{\partial \xi^{3}}\right) \\
& \frac{S^{\prime \prime}}{P r_{L}}+(2+\xi) F S^{\prime}+\xi(1-\xi)\left(P S^{\prime}-2 P^{\prime} S+2 R Q^{\prime}-R^{\prime} Q\right) \\
& \quad-2(1-2 \xi)\left(F^{\prime} S+P^{\prime} Q\right)+2 F Q^{\prime}+2(3-\xi) P Q^{\prime}+2 F^{\prime} Q \\
& \quad+(4-3 \xi) R \theta^{\prime}=\xi(1-\xi)\left(F^{\prime} \frac{\partial^{3} \theta}{\partial \xi^{3}}-\theta^{\prime} \frac{\partial^{3} F}{\partial \xi^{3}}\right) \\
& r^{\prime \prime \prime}+(2+\xi) f r^{\prime \prime}+\xi(1-\xi) p r^{\prime \prime}-2 f^{\prime} r^{\prime}-3 \xi(1-\xi) p^{\prime} r^{\prime} \\
& \quad+(4-3 \xi) f^{\prime \prime} r+2 \xi(1-\xi) p^{\prime \prime} r+2 f p^{\prime \prime}+2(3-\xi) p p^{\prime \prime} \\
& \quad-6 f^{\prime} p^{\prime}-2\left(p^{\prime}\right)^{2}=\xi(1-\xi)\left(f^{\prime} \frac{\partial^{3} f^{\prime}}{\partial \xi^{3}}-f^{\prime \prime} \frac{\partial^{3} f}{\partial \xi^{3}}\right)
\end{aligned}
$$

where the third-order differentiations with respect to $\xi$ on the right-hand side are truncated in the third-level approximation of the local nonsimilarity method.
When $T_{\infty}, u_{\infty}, T_{w}$ are prescribed for the condensation process, the heat transfer characteristics and the thermal-dynamic as well as transport properties should be determined. The current formulation assumes constant properties. Assuming $\rho_{v} \ll \rho_{L}$, the iterative steps for solving Eqs. 5, 6 and 10 with the aim to determine heat transfer at the wall expressed by Eq. 20 are presented herein for the given mixed convection parameter $\xi$ as:

I. Assume initial values respectively for $\eta_{i}$ and $F_{i}^{\prime \prime}$ which will be iteratively updated;

II. In the first-level approximation of the local nonsimilarity method, with the assumed $F_{i}^{\prime \prime}$, only two additional closure equations are needed to solve Eq. 5: they are Eqs. 14 and 15; two closure equations are needed to solve Eq. 6: they are Eqs. 16 and 17; three closure equations are needed to solve Eq. 10: they are (12), (13) and (18); the remaining two closure equations are used to update $\eta_{i}$ and $F_{i}^{\prime \prime}$;

III. In the second-level approximation, the auxiliary equations in (24) are solved together with Eqs. 5, 6 and 10; the closure equations for Eq. 24 can be derived by differentiating both sides of the closure equations in Step II with respect to $\xi$;

IV. In the third-level approximation, the auxiliary equations in (25) are solved together with Eqs. 5, 6 and 10, as well as (24); the closure equations for Eq. 25 can be derived by differentiating both sides of the closure equations in Step III with respect to $\xi$;

V. A routine ODE solver for boundary value problems is applied to the ODEs for the liquid and vapor phases; once the equations are solved, the respective temporary values are known for $f_{i}^{\prime}$ and $F_{i}^{\prime}$, which can be used by one remaining closure equation in (11) to update $F_{i}^{\prime \prime}$; another remaining closure equation in (19) is used to update $\eta_{i}$ by making use of the respective temporary values for the involved variables;

VI. Repeat the above steps until both $\eta_{i}$ and $F_{i}^{\prime \prime}$ converge; the heat transfer result is obtained by substituting into Eq. 20 the converged solution of $\theta_{0}^{\prime}$.

\section{Results}

Numerical heat transfer results from the current method using various levels of approximation are listed in the fourth to sixth columns of Table 1 . The second and thirdlevels of approximation produced results very close to each other, with the maximum relative error less than about $1 \%$. The first-level approximation produced results noticeably smaller than the second-level approximation, with the maximum relative error close to $10 \%$. Therefore, the local nonsimilarity method with the second-level approximation 
is considered sufficiently accurate and used to obtain numerical heat transfer results in the following subsections.

\subsection{Comparison to previous studies}

Numerical heat transfer results for $P r_{L}=10, \omega=10$ and a wide range of $H$ obtained using the current method were compared to those obtained by Shu and Wilks [12] and Fujii's correlation [4] in Table 1, where $z=g x / u_{\infty}^{2}$ is the mixed convection parameter used by Fujii, while $\xi=$ $1 /\left(1+z^{-1 / 4}\right)$ is the mixed convection parameter used in the current work. Numerical data coming from Shu's work (Tables 8 and 9 in [12]) were listed in Table 1 for comparison. Table 1 shows that, for smaller condensation numbers $(H)$, the discrepancy of heat transfer results produced by different methods is insignificant, while for larger condensation numbers, Fujii's correlation [4] deviates from numerical data produced by the current method and Shu's significantly, with the maximum relative error as large as $30 \%$ for those cases shown. Such discrepancy is mainly due to the neglected liquid energy convection in Fujii's correlation [4] derived from the momentum integral approach.

Additional comparison was carried out in Fig. 1 showing numerical data for a small condensation number
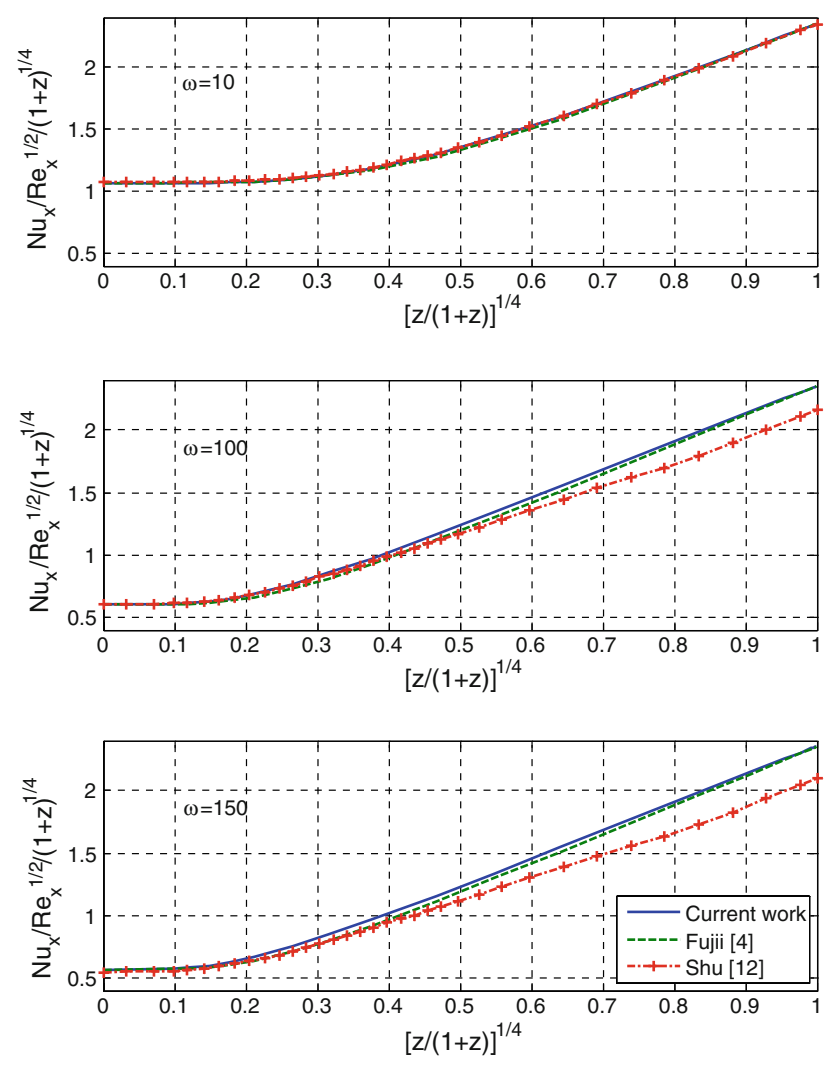

Fig. 1 Comparison of heat transfer numerical results for the entire mixed convection regime: $P r_{L}=1, H=0.008191$
$(H=0.008191)$ and for three cases with different property ratios $(\omega=10,100$ and 150$)$. Numerical data from Shu's work (Figure 5 of [12]) was duplicated here. Figure 1 shows that with a small condensation number, Fujii's correlation [4] is in good agreement with the current results for the entire mixed convection regime and for all cases with different property ratios, which implies that the liquid energy convection effect is negligibly small for condensation with a small condensation number.

Next attention is paid to the effect of the property ratio $(\omega)$ on condensation heat transfer. Both the current results and Fujii' correlation [4] show that the property ratio $(\omega)$ for free convection $\left([z /(1+z)]^{1 / 4}=1\right)$ condensation has a negligible effect on the Nusselt number. Koh et al. [9] also concluded that the property ratio $(\omega)$ has a negligible effect on the free convection Nusselt number even when $\omega$ varies from 10 to 600 , a range much wider than that in Fig. 1. The effect of the property ratio on free convection is not as strong as on forced convection because in free convection the gravity driven liquid film only induces a weak secondary flow in the vapor phase and therefore the momentum coupling of the liquid and gas phases is weaker for the free convection regime. However, Shu and Wilks's [12] data in Fig. 1 shows a significant effect of the property ratio on free convection, when comparing the Nusselt number at $[z /(1+z)]^{1 / 4}=1$ for three cases: $\omega=10,100$ and 150 . It is the authors' belief that Shu and Wilks's [12] mixed convection solution should have had the limiting case on free convection coincident with Koh et al.'s results [9] as well as those in the current work.

The comparisons of the current method to other studies demonstrate that the current one is reliable and has a high level of accuracy. Next, numerical results obtained from the current method are used to extend the applicability of Fujii's correlation [4].

\subsection{A correction factor for Fujii's correlation}

Fujii's correlation is derived from the boundary layer integral method neglecting the liquid inertia force and energy convection, and therefore the liquid Prandtl number indicating energy convection disappears in the correlation [4]:

$\frac{N u_{x}}{R e_{x}^{1 / 2}}=K\left(1+\alpha \frac{1}{4 K^{4}} \frac{z}{H}\right)^{1 / 4}, \quad K=0.45\left(1.2+\alpha \frac{1}{\omega H}\right)^{1 / 3}$

where $\alpha$ is the correction factor for the liquid energy convection effect introduced in the current work which will be defined later. When $\alpha=1$, Eq. 26 is Fujii's correlation for mixed convection laminar film condensation. 
The Nusselt number in (26) should depend not only on $z$, $H, \omega$, but also on $P r_{L}$ if the energy convection effect is taken into account. Therefore, the correction factor $\alpha$ is a function of $\operatorname{Pr}_{L}$ and other parameters. Figures 2 and 3 compare the current nonsimilarity solution with Fujii's correlation under various conditions for the purpose to evaluate the neglected energy convection effect in Fujii's correlation [4]. The following observation is drawn from reading Figs. 2 and 3. For $P r_{L}=1$ the nonsimilarity solution almost coincides with Fujii's correlation [4], indicating negligible energy convection for small $\operatorname{Pr}_{L}$. When $\operatorname{Pr}_{L}$ is gradually larger than 1, the nonsimilarity solution gradually deviates from Fujii's correlation [4], and the magnitude of deviation is augmented by the larger condensation number $(H)$, indicating significant energy convection for larger $\operatorname{Pr}_{L}$ and $H$. If other things are identical, the effect of $\omega$ is negligibly small in the regime close to free convection, but appreciable in the regime close to forced convection. Based on the above observation, the correction factor $\alpha$ must be a function of $\operatorname{Pr}_{L}, H, \omega$ and the mixed convection parameter. For the three regimes of mixed convection, the correction factor $\alpha$ is proposed below by fitting the data in the comparison between the current nonsimilarity solution and Fujii's correlation [4].

- Free convection regime $(\xi=1)$

$\alpha_{1}=1+0.68\left(P r_{L}-1\right) H$
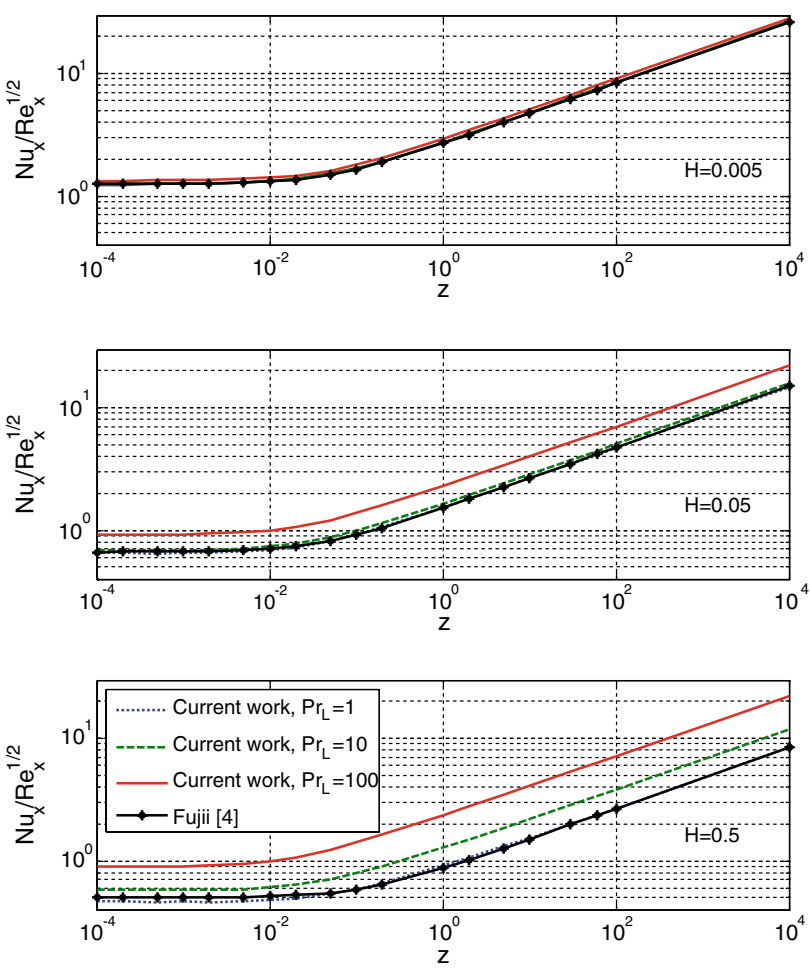

Fig. 2 Comparison of local nonsimilarity solution with Fujii's correlation [4]: $\omega=10$
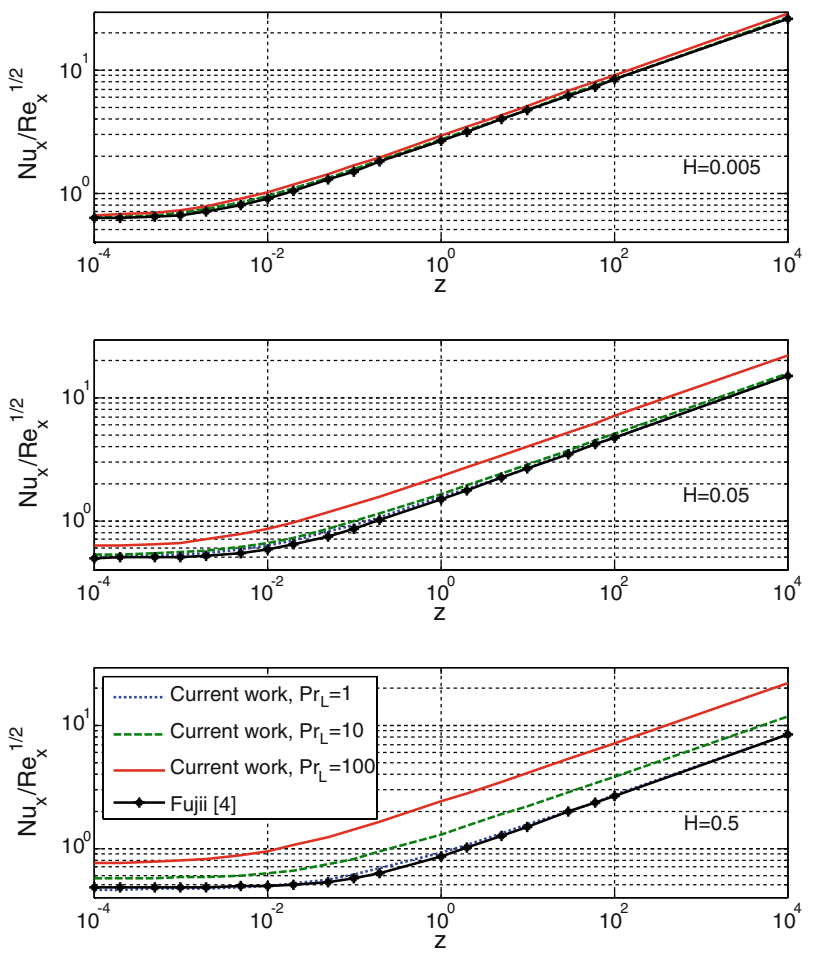

Fig. 3 Comparison of local nonsimilarity solution with Fujii's correlation [4]: $\omega=150$

which is equivalent to the Rohsenow correction factor [5] when $\operatorname{Pr}_{L} \gg$ 1. Calculation results from Eq. 26, using the correction factor in Eq. 27 to replace $\alpha$, deviate from the nonsimilarity solution with a maximum relative error of about $1 \%$ for $\operatorname{Pr}_{L}<10$ and $H<0.05$, and about $6 \%$ for $\operatorname{Pr}_{L}<100$ and $H<0.5$.

- Forced convection regime $(\xi=0)$

$\alpha_{0}=\left[1+0.55\left(\operatorname{Pr}_{L}-3\right) H\right](1+0.14 \omega H)$

Results from Eq. 26 using the correction factor in Eq. 28 deviate from the nonsimilarity solution with a maximum relative error of about $2 \%$ for $P r_{L}<10$ and $H<0.05$, and about 7\% for $\operatorname{Pr}_{L}<100$ and $H<0.5$.

- Mixed convection regime $(0<\xi<1)$

The proposed correction factor for the mixed convection regime is a weighted average of the correction factors $\alpha_{1}$ and $\alpha_{0}$ :

$\alpha=\xi \alpha_{1}+(1-\xi) \alpha_{0}$

Results from Eq. 26 using the correction factor in Eq. 29 deviate from the nonsimilarity solution with a maximum relative error of about $4 \%$ for $P r_{L}<10$ and $H<0.05$, and about $10 \%$ for $\operatorname{Pr}_{L}<100$ and $H<0.5$.

Figure 4 compares the nonsimilarity solution with Fujii's correlation [4], as well as Fujii's correlation corrected by accounting for the energy convection effect using 


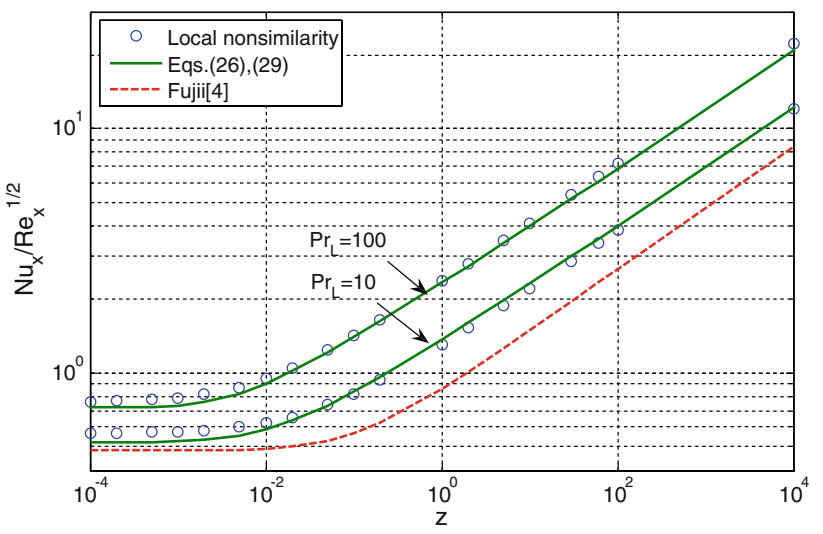

Fig. 4 Comparison of local nonsimilarity solution with Fujii's correlation [4] and the current proposed correlation using Eqs. 26 and 29: $H=0.5, \omega=100$

Eq. 29 for the full spectrum of mixed convection laminar film condensation. The comparison shows that the current correction factor can be used with Fujii's correlation [4] to predict mixed convection condensation heat transfer with significant liquid energy convection in a reasonably accurate way.

\section{Conclusions}

The current work presents a local nonsimilarity method for the two-phase boundary layer equations of laminar film condensation. The analysis treats the full spectrum of mixed convection, with a generic boundary layer formulation which was demonstrated herein to reduce to two specific cases mathematically identical to Koh's formulations on the two limiting cases of either free or forced convection. In between, the solution for mixed convection condensation was carried out using different levels of approximation to investigate the asymptotical behavior of the local nonsimilarity method. It was observed that the second-level approximation has the optimum trade-off between the level of accuracy achieved and the amount of effort spent on the formulation. Other solution methods for mixed convection in the literature were compared and critically evaluated to ensure a high level of accuracy in the current local nonsimilarity method. The current solution is used to extend Fujii's correlation for mixed convection laminar film condensation to the region where the energy convection effect is significant but has been hitherto neglected. The modified Fujii correlation depends on the temperatures, bulk velocity and fluid properties only, which is convenient to be applied to a wide variety of engineering applications.

\section{References}

1. Nusselt W (1916) Die Oberflachenkondensation des Wasserdampfes. Z Vereines Deutsch Ing 60:541-546, 569-575

2. Fujii T (1991) Theory of laminar film condensation. SpringerVerlag, New York

3. Rose JW (1999) Condensation heat transfer. Heat Mass Transfer 35:479-485

4. Fujii T, Uehara H (1972) Laminar filmwise condensation on a vertical surface. Int J Heat Mass Transf 15:217-233

5. Rohsenow WM (1956) Heat transfer and temperature distribution in laminar-film condensation. Trans ASME 78:1645-1648

6. Chen MM (1961) An analytical study of laminar film condensation. Part 1: flat plates and part 2 multiple horizontal tubes. ASME J Heat Transf 83:48-60

7. Sadasivan P, Lienhard JH (1987) Sensible heat correction in laminar film boiling and condensation. ASME J Heat Transf 109:545-547

8. Sparrow EM, Gregg JL (1956) A boundary-layer treatment of laminar-film condensation. ASME J Heat Transf 81:13-18

9. Koh JCY, Sparrow EM, Hartnett JP (1961) The two phase boundary layer in laminar film condensation. Int $\mathrm{J}$ Heat Mass Transf 2:69-82

10. Koh JCY (1962) Film condensation in a forced-convection boundary-layer flow. Int J Heat Mass Transf 5:941-954

11. Denny VE, Mills AF (1969) Nonsimilar solutions for laminar film condensation on a vertical surface. Int $\mathrm{J}$ Heat Mass Transf 12:965-979

12. Shu J, Wilks G (1995) Mixed-convection laminar film condensation on a semi-infinite vertical plate. J Fluid Mech 300:207-229

13. Winkler CM, Chen TS (2000) Mixed convection in film condensation from isothermal vertical surfaces-the entire regime. Int J Heat Mass Transf 43:3245-3251

14. Sparrow EM, Yu HS (1971) Local nonsimilarity thermal boundary layer solutions. ASME J Heat Transf 93:328-334 